\title{
GM-CSF and the role of myeloid regulatory cells in the pathogenesis and treatment of Crohn's disease
}

\author{
Jan Däbritz ${ }^{1,2,3}$
}

\begin{abstract}
Background: Intestinal monocytes/macrophages sustain the intestinal immune homeostasis and might be an attractive therapeutic target for the management of inflammatory bowel disease (IBD). Granulocyte macrophage colony-stimulating factor (GM-CSF) exerts beneficial effects on intestinal inflammation and promotes signal transducer and activator of transcription 3 (STAT3)-mediated expansion of myeloid-derived suppressor cells (MDSCs). However, the full action mechanism of GM-CSF, and especially whether monocytes mediate its therapeutic effects in vivo, had not been previously elucidated.

Conclusions: This review article summarizes recent developments in the immunology of mucosal diseases and describes new aspects of the role of myeloid regulatory cells in IBD and the function of GM-CSF in maintaining the intestinal immune homeostasis in Crohn's disease (CD). This review article highlights the exploration of stimulating in addition to suppressive therapies for patients with IBD and underpins that myeloid regulatory cells might become a promising novel cell-based therapeutic option.
\end{abstract}

\section{Introduction}

Crohn's disease (CD) is a chronic relapsing disease and is a manifestation of a dysregulated immune response against the microorganisms of the intestinal flora in genetically susceptible individuals [1]. Various components of the mucosal immune system are implicated in the pathogenesis of inflammatory bowel diseases (IBD) and include intestinal epithelial cells; innate lymphoid cells; cells of the innate immune system such as monocytes, macrophages, neutrophils, and dendritic cells (DCs); the adaptive immune system with $\mathrm{T}$ and $\mathrm{B}$ cells; and their secretion products (chemokines, cytokines) (Fig. 1). Investigating the microbiome will come up with new theories on the etiology of IBD. Thus, the increasing knowledge of the interaction between the innate and adaptive immune systems as well as the microbiome in the context of the individual genetic background might be helpful for personalized therapy in IBD in the future. Indeed,

\footnotetext{
Correspondence: jan.daebritz@med.uni-rostock.de

${ }^{1}$ Present address: Department of Pediatrics, University Hospital Rostock, Ernst-Heydemann-Str. 8, 18057 Rostock, Germany

${ }^{2}$ Department of Pediatric Rheumatology and Immunology, University Hospital Münster, Albert-Schweitzer-Campus 1, 48149 Münster, Germany Full list of author information is available at the end of the article
}

the intestinal mucosa is host to a massive number of intestinal macrophages resident in the mucosa which continuously demonstrate phagocytic activity, and blood monocytes are recruited to the lamina propria, normally quickly undergo induction of anergy but interestingly retain many proinflammatory activities in inflamed mucosa. Although the exact cause of $\mathrm{CD}$ remains unknown, new pathogenic paradigms in IBD have highlighted that the interactions between various constituents of the innate and adaptive immune systems play key roles in the pathogenesis of IBD [2]. As the frontiers of immunological research expand, new insights into the pathogenesis of IBD are opening up new possible avenues for treatment. Myeloid-derived cells including monocytes/macrophages long thought to be effector cells driving the initiation of inflammation have been increasingly shown to have immunoregulatory effects previously under-appreciated. Recent work suggests an important protective role of monocytes/macrophages and possible homeostatic mechanisms to restrain acute and chronic intestinal inflammation (reviewed in [3]). This brief review highlights the important role of immunoregulatory monocytes in IBD and the role of granulocyte macrophage colony-stimulating factor (GM-CSF) in maintaining the

\section{Springer}




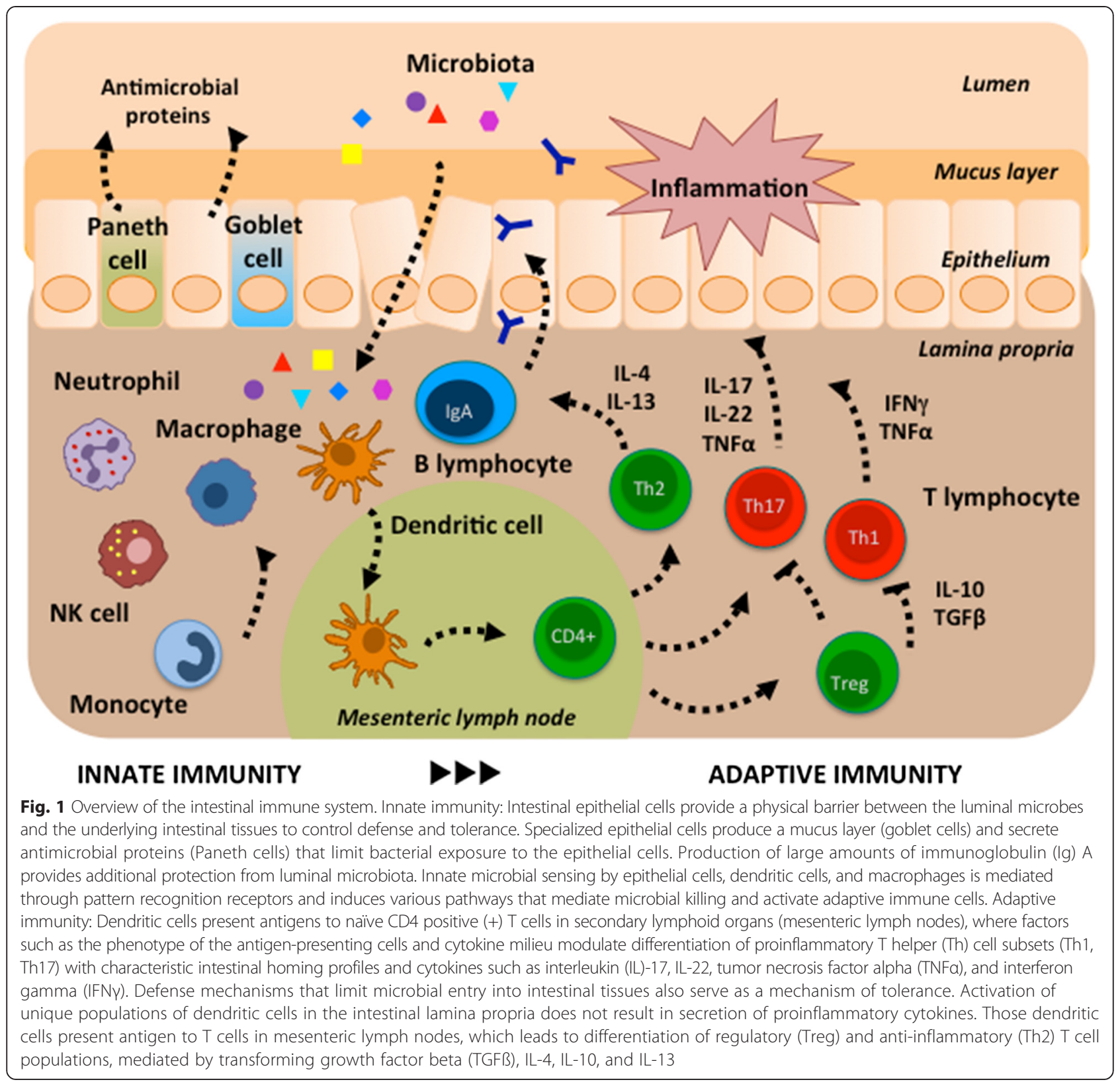

intestinal immune homeostasis in CD. For earlier and more detailed literature on the immunoregulatory role of myeloid-derived cells in IBD, the reader is referred to prior reviews ([3] and references cited therein).

\section{Role of monocytes/macrophages and GM-CSF in the immunology of mucosal diseases}

Monocytes and their derivative cells play an important role in the pathophysiology of IBD. Intestinal macrophages derived from blood monocytes play a key role in sustaining the innate immune homeostasis in the intestine [4]. Intestinal chronic inflammation may be caused by inborn errors of macrophages in patients with CD. Macrophages from CD patients are intrinsically defective, with impaired secretion of cytokines that are normally translated but internally degraded. Because of insufficient production of cytokines and chemokines, there is impaired attraction of granulocytes to mucosal breaches. Impaired acute, granulocytic inflammation results in impaired clearance of bacteria and debris from the gut wall, itself resulting in chronic, granulomatous inflammation $[5,6]$. The cytokine GM-CSF promotes the differentiation of monocytes into macrophages and DCs. There is growing evidence that GM-CSF, or rather the lack thereof, is implicated in the pathogenesis of $\mathrm{CD}$. Human and murine studies showed that GM-CSF exerts beneficial effects on intestinal inflammation. In addition, it has been shown that increased levels of neutralizing 
endogenous serum GM-CSF autoantibodies $(\mathrm{Ab})$ in $\mathrm{CD}$ patients as well as decreased expression of the GM-CSF receptor $\alpha$-subunit (CD116) in circulating monocytes and granulocytes of $\mathrm{CD}$ patients affect mucosal integrity, epithelial barrier function, bacterial killing, and neutrophil antimicrobial functions (reviewed in [7]).

GM-CSF also triggers several different signaling pathways in myeloid-derived suppressor cells (MDSCs) that mainly involve the signal transducer and activator of transcription (STAT) family of transcription factors. MDSCs are a heterogeneous group of immature cells that includes precursors of macrophages, granulocytes, DCs, and myeloid cells at earlier stages of differentiation. MDSCs expand rapidly during inflammation, infection, and cancer and suppress T cell responses [8]. Although MDSCs have been described in animal models of experimental colitis and in patients with IBD, their exact role in IBD pathogenesis is unclear [9]. As an additional homeostatic function, GM-CSF has been proposed to be essential for a microbiota-dependent crosstalk between mononuclear phagocytes and group 3 innate lymphoid cells (ILC3s) thereby promoting intestinal homeostasis. ILC3derived GM-CSF can promote intestinal myeloid cell homeostasis through enhancing DC and regulatory $\mathrm{T}$ cell function [10-13].

The potential for future cell-based therapies for IBD is enhanced by the advances being made in the understanding of the innate immune system in the intestine. Based on this, any therapeutic approach aimed at modulating monocytes/macrophages as a key player in innate immunity could be a potent way to modulate/manage intestinal inflammation in patients with chronic intestinal inflammation. However, the full action mechanism of GM-CSF, and especially whether monocytes mediate its therapeutic effects in vivo, had not been previously elucidated.

\section{Human GM-CSF-activated monocytes have regulatory immune functions in vitro}

We hypothesized that GM-CSF might activate monocytes in a way that modulates their function during intestinal inflammation. We analyzed the immunoregulatory potential of GM-CSF on human monocytes in vitro using microarray technology and functional assays taking all potential functions of human GM-CSF-activated monocytes into account (including gene expression, innate immune functions, interplay with adaptive immunity, wound healing). Our findings suggest that the early imprinting of monocytes after activation with GM-CSF is of crucial importance, because monocytes play an important role during the recruitment phase of the innate immune response and have the potential to regulate adaptive immune mechanisms.
We showed that GM-CSF-activated monocytes combine (i) an anti-inflammatory phenotype, (ii) features of augmented innate immune functions (e.g., migration, chemotaxis, oxidative burst), (iii) the ability to facilitate epithelial healing, and (iv) the regulatory potential on adaptive immunity. GM-CSF-activated monocytes represent an intermediate cell type, combining cell-surface expression characteristics and functional features of different M2-type macrophage subsets [14]. In contrast with proinflammatory and antimicrobial responses of classically activated M1-like monocytes, M2-like phenotypes are broadly anti-inflammatory and play important roles in wound healing [15]. In addition to these innate immune functions, human GM-CSF-activated monocytes simultaneously have a regulatory potential on adaptive immunity. We showed that GM-CSF significantly induces a short-termed expression of chemokines in monocytes, which are known to attract naïve $\mathrm{T}$ cells, $\mathrm{T}$ helper 2 (Th2) cells, and/or regulatory $\mathrm{T}$ cells (Treg). Indeed, migration of naïve, autologous T cells toward GM-CSFactivated monocytes was accelerated in vitro. Our data suggest that particularly CCL18 and CCL 23 might be responsible for the increased $\mathrm{T}$ cell migration. When we cocultured GM-CSF-activated monocytes with syngeneic $\mathrm{CD}^{+} \mathrm{T}$ cells, we observed that GM-CSF-activated monocytes attract $\mathrm{T}$ cells and shape their differentiation toward Th2 cells by upregulating T cell-derived IL-4, IL-10, and IL-13 and downregulating T cell-derived IFN $\gamma$ in vitro. In addition, cocultures of GM-CSFactivated monocytes and naïve $\mathrm{T}$ cells led to an induction of Treg, which produce anti-inflammatory IL-10 and TGF $\beta$ and have important anti-inflammatory functions [14].

Finally, we showed that GM-CSF may regulate the homing molecules CCR2 and CCR6 on human monocytes [14], which are involved in regulating several aspects of mucosal immunity, including the ability to mediate the recruitment of innate immune cells to the sites of epithelial inflammation.

\section{Murine GM-CSF-activated monocytes as an anti- inflammatory player in the intestine}

After having analyzed the specific activation pattern of human monocytes in response to GM-CSF in vitro, we next aimed to address the functionality of these cells in vivo in the context of $\mathrm{CD}$. Because systematic analyses in the human system are not feasible, we went on analyzing the effects of GM-CSF-activated monocytes on the murine system. In the first step, we activated bonemarrow-derived murine monocytes with GM-CSF in vitro and analyzed the phenotype and functional properties of murine GM-CSF-activated monocytes in vitro.

Our gene expression analyses showed that murine GM-CSF-activated monocytes have a similar gene 
regulation profile when compared with the GM-CSFdependent gene expression in human monocytes [14]. Like human GM-CSF-activated monocytes, murine GMCSF-activated monocytes did not fit into current monocyte subtype definitions dividing monocytes/macrophages according to cell surface markers and functions in classic/ inflammatory (M1), intermediate, and alternative/anti-inflammatory (M2) cells. After activation with GM-CSF, murine monocytes were found to overexpress some surface markers of specialized macrophages and some stimulatory molecules while showing decreased expression of a marker for alternatively activated monocytes. In addition, we noted discrepancies in the expression levels of some M2-polarized markers. Because this expression profile did not fully recapitulate the previously reported activation stages of monocytes, we proposed that the newly generated monocytes represented a unique and distinct state of activation. These murine GM-CSF-activated monocytes were also found to be somewhat unique from a functional point of view. We demonstrated in vitro that this population has a lower capacity for phagocytosis and adhesion but triggers increased generation of reactive oxygen species, which are required for pathogen clearance. Moreover, after lipopolysaccharide treatment, proinflammatory cytokine production was found to be higher in GM-CSFactivated monocytes compared with untreated monocytes. However, despite their phenotypical and functional diversity, murine GM-CSF-activated monocytes mainly seem to represent monocytes in a developmental stage passing toward M2-polarized macrophages with an upregulation of M2 markers and important anti-inflammatory functions [16].

In addition, we performed flow cytometry-based staining of various gut homing molecules on monocytes and found, in agreement with our studies on human GMCSF-activated monocytes, CCR2, necessary for migration toward inflammatory sites, and CCR6, used for the migration toward lymph follicles such as Peyer patches, to be upregulated on murine GM-CSF-activated monocytes [16].

\section{Murine GM-CSF-activated monocytes reduce se- verity of experimental colitis}

In the next step, we tested whether GM-CSF-activated monocytes have a protective and therapeutic effect against intestinal inflammation in vivo. Since none of the currently available animal models of IBD reflects all aspects of IBD in humans, we used various mouse models of chronic colitis. Firstly, we showed that Rag1deficient mice (which lack all mature adaptive immune cells) were protected from disease progression during $\mathrm{CD} 4{ }^{+} \mathrm{CD} 25^{-} \mathrm{T}$ cell-induced experimental colitis when they received GM-CSF-activated monocytes but not untreated monocytes. In agreement with earlier work, we found that Rag1-deficient mice that did not receive GMCSF-activated monocytes but GM-CSF intraperitoneally after the transfer of $\mathrm{T}$ cells were not completely protected from colitis but showed reduced disease severity. Our data demonstrate that the protective effect of monocytes depends on their GM-CSF pre-activation in a $T$ cell-dependent model of colitis [14]. Secondly, we induced chronic colitis in wild-type mice by repetitive administration of dextran sulfate sodium (DSS) in drinking water. Mice treated with GM-CSF-activated monocytes were again completely protected from experimental colitis [16]. Thirdly, we induced chronic colitis in Rag1deficient mice with DSS. Injection of GM-CSF-activated monocytes was not able to protect Rag1-deficient mice against DSS-induced colitis, indicating that adaptive immunity is required for the protection mediated by GMCSF-activated monocytes [16].

We next sought to decipher the mechanism by which GM-CSF-activated monocytes confer their therapeutic effects on intestinal inflammation. We found that, compared with control monocytes, the GM-CSF-activated monocytes were taken up faster by the inflamed intestine and stayed there longer, especially in Peyer patches $[14,16]$. In addition to these unique migratory features and in agreement with our in vitro findings, accelerated gut homing of GM-CSF-activated monocytes was accompanied by increased production of the anti-inflammatory Th2 cytokines IL-4, IL-10, and IL-13 and decreased production of the proinflammatory Th1 cytokine IFN $\gamma$ in intestinal lamina propria mononuclear cells and mesenteric lymph nodes in vivo during colitis [14]. Furthermore, GM-CSF-activated monocytes increased the accumulation of Foxp $3^{+}$Tregs in the lymph follicles of mice with experimental colitis when compared with untreated mice or those treated with nonactivated monocytes. In an in vitro model in which GM-CSF-activated monocytes were cocultured with naïve $\mathrm{T}$ cells, we observed increased proliferation and differentiation of Foxp $3^{+}$Treg cells and found that this occurred through a mechanism that involves the CD39-mediated conversion of ATP into adenosine-a mechanism that was previously reported to induce Treg cell differentiation [16].

Taken together, these important data show that GMCSF-activated monocytes are a specific population of monocytes that has a protective effect against intestinal inflammation (Fig. 2). The beneficial effects of GM-CSFactivated monocytes are $\mathrm{T}$ cell dependent and involve the CD39/adenosine-mediated proliferation and differentiation of Foxp3 ${ }^{+}$Treg cells. This is in agreement with the work of others, who showed that GM-CSF can influence the interactions between DCs and T cells and in this manner determine a pro- versus anti-inflammatory adaptive immune response via Th1 and Th17 cell responses or the induction of Treg cells, respectively [17-20]. 


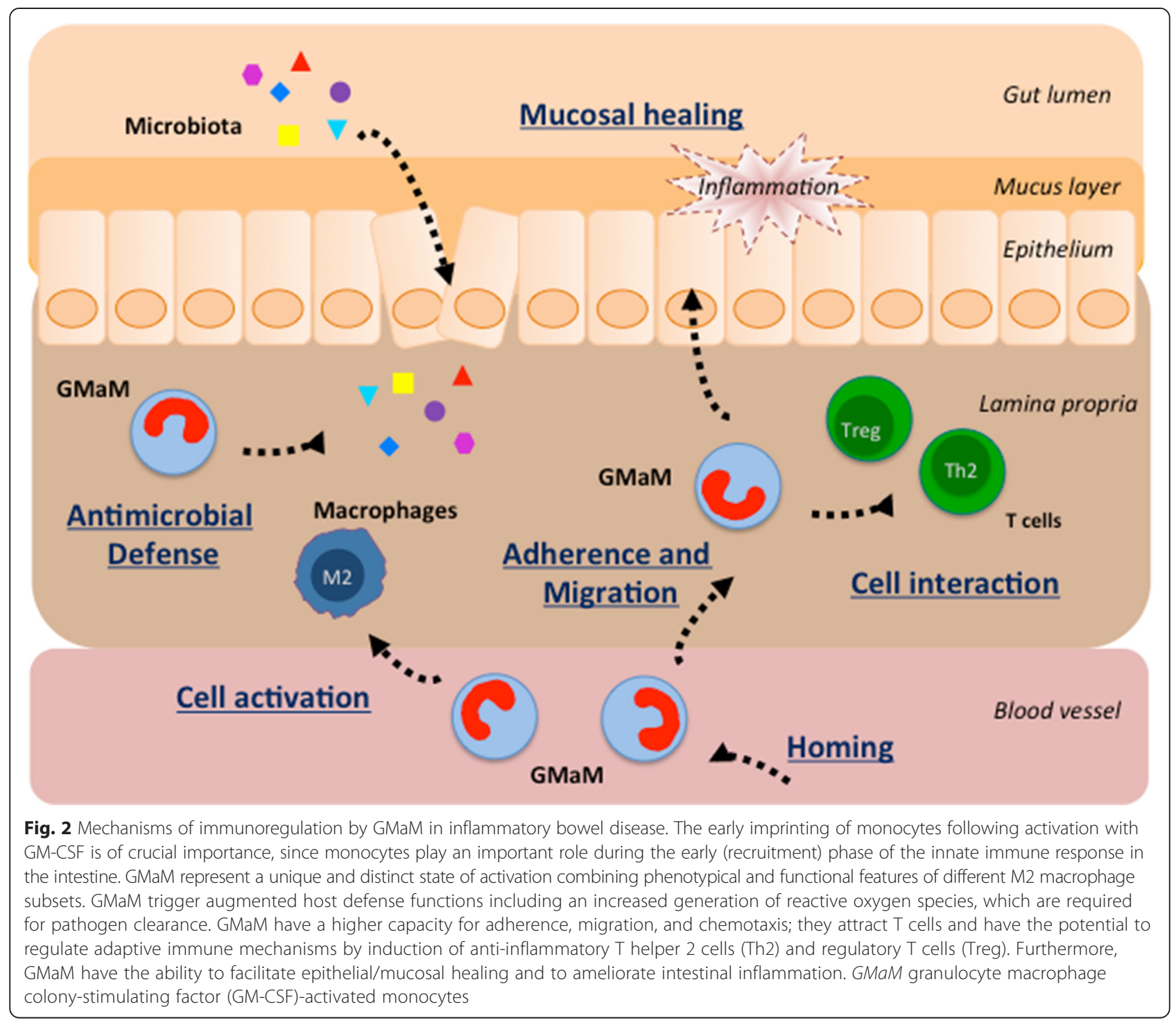

\section{Neutralization of GM-CSF is associated with dis- ease relapse in $C D$ patients}

GM-CSF bioactivity varies inversely with increasing serum levels of endogenous neutralizing GM-CSF autoantibodies (Ab). GM-CSF Ab as well as defective GMCSF receptor expression have been described in IBD [21-25]. Increased GM-CSF Ab levels are associated with alterations of epithelial barrier function, bowel permeability, bacterial translocation, neutrophil antimicrobial functions, and epithelial cell survival and proliferation [7, 21-24, 26, 27]. A rare familial form of pulmonary alveolar proteinoses may also serve as an established model to highlight the role of Ab toward GM-CSF resulting in blocking GM-CSF-driven responses [28, 29]. This suggested that variation in GM-CSF Ab and thereby GMCSF bioactivity might also be associated with clinical disease relapses in patients with $C D$. In order to validate these findings, we determined GM-CSF Ab levels in prospectively collected serum samples of pediatric and adult patients with CD. Time course analysis of GM$\mathrm{CSF} \mathrm{Ab}$ up to 9 months before and after relapse showed a clear increase of $\mathrm{Ab}$ titers up to 6 months before clinical relapse followed by a steady decrease, likely indicating the success of the intensified therapies. In addition, we found that GM-CSF Ab levels in serum samples of patients with IBD who had no clinical disease relapse during the study follow-up showed a low intra-individual variation and GM-CSF Ab levels were below the cut-point where GM-CSF Ab start to inhibit neutrophil antibacterial function [30]. Thus, an elevated serum GM-CSF Ab titer in patients with $C D$ in clinical remission as defined by clinical disease activity scores may represent an early stage of increased intestinal permeability, bacterial translocation, neutrophil dysfunction, and reduced antimicrobial activity (reviewed in [31]). It is conceivable that the potentially impaired intestinal homeostasis progresses to 
cause an eventual clinical relapse of the disease and underpins the beneficial role of GM-CSF in the context of intestinal homeostasis.

\section{Blood monocytes from patients with CD behave like GM-CSF-activated monocytes}

Our phenotypic and functional studies showed that peripheral blood monocytes from CD patients in clinical remission are not impaired compared to healthy controls [32]. We next studied phenotypic and functional features of untreated versus GM-CSF-activated peripheral blood monocytes of patients with quiescent $\mathrm{CD}$. Collectively, our data suggest that the effects of GM-CSF activation of peripheral blood monocytes from patients with $\mathrm{CD}$ are similar to the observed effects of GM-CSF-activated monocytes from healthy donors. This included the GM-CSF-induced increase in adherence, migration, chemotaxis, and oxidative burst, as well as the priming of monocytes to secondary microbial stimuli. In addition, changes in GM-CSF-dependent mRNA expression of selected key inflammatory cytokines were in agreement with our transcriptomic data obtained from GM-CSFactivated monocytes of healthy individuals. Importantly, there was no evidence that GM-CSF activation had different effects on monocytes when compared between individual CD patients [14].

\section{Myeloid-specific STAT3 activation ameliorates experimental colitis via MDSCs}

Several studies have investigated the mechanisms of MDSC $\mathrm{T}$ cell suppressive activity in health and disease including experimental colitis models and human IBD $[8,9]$. MDSC expansion is regulated by several factors (including GM-CSF) that are released during inflammation, infection, and cancer. The signaling pathways in MDSCs converge on Janus kinase (JAK) protein family members and STAT3. Hyperactivation of STAT3 in gp130 ${ }^{757 \mathrm{~F} / \mathrm{F}}$ mice is associated with protection from DSS-induced colitis. We hypothesized that the protective role of gp130-dependent STAT3 activation in experimental IBD involves the expansion and activation of MDSCs, in addition to the previously reported proliferative, regenerative, and survival effects on intestinal epithelial cells. We therefore induced acute and also chronic colitis with DSS and analyzed the effects in mice with systemic hyperactivation of STAT3 $\left(\mathrm{gp} 130^{757 \mathrm{~F} / \mathrm{F}}\right) \pm$ myeloid-specific STAT3-deficiency (LysMcre/STAT3 ${ }^{\text {flox }}$ ). We showed that the resistance to DSS-induced acute colitis in gp130 $757 \mathrm{~F} / \mathrm{F}$ mice is via myeloid cell-specific STAT3 activation, expansion of granulocytic MDSCs in the colon, and increased production of suppressive and protective mediators. Interestingly, gp130 $137 \mathrm{~F} / \mathrm{F}$ mice were not resistant to chronic DSS-induced colitis [33].
Thus, our study identified new immunoregulatory mechanisms of STAT3 during intestinal inflammation. Importantly, the adoptive transfer of MDSCs in different animal models of IBD ameliorates colitis and suggests that MDSCs may be used as the basis for a novel adoptive cellular cell therapy in IBD ([3] and references cited therein). However, MDSCs may either promote or suppress $\mathrm{T}$ cell functions depending on the stage of disease and/or other external factors. Indeed, there is also evidence supporting a proinflammatory role of myeloid cells in experimental IBD [34-36]. In addition, an increase in the frequency of MDSCs in the peripheral blood from patients with active chronic colitis has been reported [37].

\section{Summary and future perspectives}

The work of others and our in vitro and in vivo data show that GM-CSF-activated monocytes are a specific population of monocytes that has a protective effect against intestinal inflammation as shown in various independent mouse models of experimental colitis. The beneficial effects of GM-CSF-activated monocytes are T cell dependent and involve the proliferation and differentiation of Th2 and Treg cells in the intestine (Fig. 2). Thus, our in vitro and in vivo findings support the exploration of stimulating rather than suppressive therapies with the potential to more specifically reprogram monocytes toward immunoregulatory functions to alleviate chronic $\mathrm{CD}$. Even though previous clinical trials showed that systemic administration of GM-CSF might have therapeutic effects in a subgroup of patients with CD (reviewed in [38]), it is important to consider that key aspects of GM-CSF biology and unwanted side effects of systemic administration still need to be clarified [39]. Adverse events commonly associated with systemic GM-CSF administration include injection site reactions, bone and musculoskeletal chest pain, pulmonary capillary leak syndrome, pulmonary edema, heart failure, fever, and neurotoxicity. In addition, in many pre-clinical models of inflammation and autoimmunity, GM-CSF neutralization or deletion suppresses a number of diseases including the following: arthritis, experimental autoimmune encephalomyelitis, lung inflammation and disease (asthma and chronic obstructive pulmonary disease), nephritis, psoriasis, atherosclerosis, cancer (e.g., breast cancer), Alzheimer's disease, myocardial infarction, peripheral insulin resistance, and inflammatory pain. Thus, beneficial effects of GM-CSF-driven tolerance in CD seem somewhat counterintuitive, as GM-CSF is generally regarded as a cytokine with more proinflammatory functions based on its activity on neutrophils and macrophages. It is likely that GM-CSF plays both protective and pathological roles in IBD and that the context in which it is produced might determine its ultimate functional role. 
Recent work, for example, showed that GM-CSF promotes IL-23-driven intestinal inflammation through local accumulation of activated eosinophils and potentiation of their effector functions [40]. A targeted cell-based approach using GM-CSF-activated monocytes represents a safer and more promising treatment option given the pleiotropic and proinflammatory effects of GM-CSF on inflammation and autoimmunity (reviewed in [41]). Given the contrasting roles for GM-CSF in immunopathology, our work helps to strengthen the link between GM-CSF and IBD [42] and is opening up new possible avenues for a personalized IBD treatment.

\section{Competing interests}

The author declares that he has no competing interests.

\section{Author's contributions}

JD conceived, wrote, and approved the manuscript.

\section{Author's information}

Dr. Däbritz is a clinician scientist with a focus on inflammatory bowel diseases (IBD). He trained in General Pediatrics and Pediatric Gastroenterology and received post-doctoral training in Mucosal Immunology at the University Children's Hospital in Münster (Germany). Dr. Däbritz then did a Pediatric Gastroenterology fellowship at the Royal Children's Hospital in Melbourne (Australia), where he undertook translational research at the Murdoch Children's Research Institute into the endogenous regulation of inflammation in IBD. Dr. Däbritz moved to the University Children's Hospital in Rostock (Germany) to become a consultant pediatric gastroenterologist, clinical senior lecturer, head of research, and deputy director at the Department of Pediatrics.

\section{Acknowledgements}

Supported by research grants awarded by the German Research Foundation (DFG DA1161/4-1, DFG DA1161/5-1), the Crohn's and Colitis Foundation of America (CCFA 1911), the Broad Medical Research Program of the Eli and Edythe Broad Foundation (BMRP IBD0201), the Innovative Medical Research Program of the University of Münster (IMF DÄ120904, IMF DÄÜ21003), and the Interdisciplinary Center for Clinical Research of the University of Münster (IZKF Fö2/005/06).

The author thanks the German Society of Pediatrics and Adolescent Medicine (DGKJ) for the Friedrich Linneweh Award 2015 and the German Society of Gastroenterology, Digestive and Metabolic Diseases (DGVS) for the Harald Goebell Award 2009. The author thanks the American Gastroenterological Association (AGA) Research Foundation for the Moti L and Kamla Rustgi Awards 2009 and 2010, which supported the presentation of the research at the 50th and 51st Digestive Disease Week annual meetings in Chicago (IL) and New Orleans (LA). The author also thanks the United European Gastroenterology (UEG) for the National Scholar Award 2013, which supported the presentation of the research at the 21st UEG Week annual meeting in Berlin (Germany).

\section{Author details}

${ }^{1}$ Present address: Department of Pediatrics, University Hospital Rostock, Ernst-Heydemann-Str. 8, 18057 Rostock, Germany. ${ }^{2}$ Department of Pediatric Rheumatology and Immunology, University Hospital Münster, Albert-Schweitzer-Campus 1, 48149 Münster, Germany. ${ }^{3}$ Murdoch Children's Research Institute, The Royal Children's Hospital Melbourne, 50 Flemington Road, Parkville, VIC 3052, Australia.

Received: 17 August 2015 Accepted: 20 November 2015 Published online: 01 December 2015

\section{References}

1. Van Limbergen J, Radford-Smith G, Satsangi J (2014) Advances in IBD genetics. Nat Rev Gastroenterol Hepatol 11(6):372-385. doi:10.1038/nrgastro.2014.27

2. Cader MZ, Kaser A (2013) Recent advances in inflammatory bowel disease: mucosal immune cells in intestinal inflammation. Gut 62(11):1653-1664. doi:10.1136/gutjnl-2012-303955
3. Leal MC, Däbritz J (2015) Immunoregulatory role of myeloid-derived cells in inflammatory bowel disease. Inflamm Bowel Dis 2015;21(12): 2936-47. doi:10.1097/MIB.0000000000000511

4. Bain CC, Mowat AM (2014) The monocyte-macrophage axis in the intestine. Cell Immunol 291(1-2):41-48. doi:10.1016/j.cellimm.2014.03.012

5. Khor B, Gardet A, Xavier RJ (2011) Genetics and pathogenesis of inflammatory bowel disease. Nature 474(7351):307-317. doi:10.1038/nature10209

6. Casanova JL, Abel L (2009) Revisiting Crohn's disease as a primary immunodeficiency of macrophages. J Exp Med 206(9):1839-1843. doi:10.1084/jem.20091683

7. Däbritz J (2014) Granulocyte macrophage colony-stimulating factor and the intestinal innate immune cell homeostasis in Crohn's disease. Am J Physiol Gastrointest Liver Physiol 306(6):G455-465. doi:10.1152/ajpgi.00409.2013

8. Gabrilovich DI, Nagaraj S (2009) Myeloid-derived suppressor cells as regulators of the immune system. Nat Rev Immunol 9(3):162-174. doi:10.1038/nri2506

9. Ostanin DV, Bhattacharya D (2013) Myeloid-derived suppressor cells in the inflammatory bowel diseases. Inflamm Bowel Dis 19(11):2468-2477. doi:10.1097/MIB.0b013e3182902b11

10. Sonnenberg GF, Artis D (2015) Innate lymphoid cells in the initiation, regulation and resolution of inflammation. Nat Med 21(7):698-708. doi:10.1038/nm.3892

11. Eberl G, Colonna M, Di Santo JP, McKenzie AN (2015) Innate lymphoid cells. Innate lymphoid cells: a new paradigm in immunology. Science 348(6237): aaa6566. doi:10.1126/science.aaa6566

12. Goldberg R, Prescott N, Lord GM, MacDonald TT, Powell N (2015) The unusual suspects - innate lymphoid cells as novel therapeutic targets in IBD. Nat Rev Gastroenterol Hepatol 12(5):271-283. doi:10.1038/nrgastro.2015.52

13. Artis D, Spits H (2015) The biology of innate lymphoid cells. Nature 517(7534):293-301. doi:10.1038/nature14189

14. Däbritz J, Weinhage T, Varga G, Wirth T, Walscheid K, Brockhausen A, Schwarzmaier D, Bruckner M, Ross M, Bettenworth D, Roth J, Ehrchen JM, Foell D (2015) Reprogramming of monocytes by GM-CSF contributes to regulatory immune functions during intestinal inflammation. J Immunol 194(5):2424-2438. doi:10.4049/jimmunol.1401482

15. Italiani P, Boraschi D (2014) From monocytes to M1/M2 macrophages: phenotypical vs. functional differentiation. Front Immunol 5:514. doi:10.3389/fimmu.2014.00514

16. Weinhage T, Däbritz J, Brockhausen A, Wirth T, Brückner M, Belz M, Foell D, Varga G (2015) Granulocyte macrophage colony-stimulating factor-activated CD39+/CD73+ murine monocytes modulate intestinal inflammation via induction of regulatory T cells. Cell Mol Gastroenterol Hepatol 1(4):433-449. doi:10.1016/j.jcmgh.2015.04.005

17. Samson CM, Jurickova I, Molden E, Schreiner W, Colliver J, Bonkowski E, Han X, Trapnell BC, Denson LA (2011) Granulocyte-macrophage colony stimulating factor blockade promotes ccr9(+) lymphocyte expansion in Nod2 deficient mice. Inflamm Bowel Dis 17(12):2443-2455. doi:10.1002/ibd.21672

18. Cheatem D, Ganesh BB, Gangi E, Vasu C, Prabhakar BS (2009) Modulation of dendritic cells using granulocyte-macrophage colony-stimulating factor (GM-CSF) delays type 1 diabetes by enhancing CD4+CD25+ regulatory T cell function. Clin Immunol 131(2):260-270. doi:10.1016/j.clim.2008.12.001

19. Ganesh BB, Cheatem DM, Sheng JR, Vasu C, Prabhakar BS (2009) GM-CSFinduced CD11c+CD8a - dendritic cells facilitate Foxp3+ and IL-10+ regulatory $T$ cell expansion resulting in suppression of autoimmune thyroiditis. Int Immunol 21(3):269-282. doi:10.1093/intimm/dxn147

20. Gaudreau S, Guindi C, Menard M, Besin G, Dupuis G, Amrani A (2007) Granulocyte-macrophage colony-stimulating factor prevents diabetes development in NOD mice by inducing tolerogenic dendritic cells that sustain the suppressive function of CD4+CD25+ regulatory T cells. J Immunol 179(6):3638-3647

21. Dykes DM, Towbin AJ, Bonkowski E, Chalk C, Bezold R, Lake K, Kim MO, Heubi JE, Trapnell BC, Podberesky DJ, Denson LA (2013) Increased prevalence of luminal narrowing and stricturing identified by enterography in pediatric Crohn's disease patients with elevated granulocyte-macrophage colony stimulating factor autoantibodies. Inflamm Bowel Dis 19(10):2146-2154. doi:10.1097/MIB.0b013e31829706e0

22. Gathungu G, Kim MO, Ferguson JP, Sharma Y, Zhang W, Ng SM, Bonkowski E, Ning K, Simms LA, Croft AR, Stempak JM, Walker N, Huang N, Xiao Y, Silverberg MS, Trapnell B, Cho JH, Radford-Smith GL, Denson LA (2013) Granulocyte-macrophage colony-stimulating factor autoantibodies: a marker of aggressive Crohn's disease. Inflamm Bowel Dis 19(8):1671-1680. doi:10.1097/MIB.0b013e318281f506 
23. Han X, Uchida K, Jurickova I, Koch D, Willson T, Samson C, Bonkowski E, Trauernicht A, Kim MO, Tomer G, Dubinsky M, Plevy S, Kugathsan S, Trapnell BC, Denson LA (2009) Granulocyte-macrophage colony-stimulating factor autoantibodies in murine ileitis and progressive ileal Crohn's disease. Gastroenterology 136(4):1261-1271. doi:10.1053/j.gastro.2008.12.046, e1261-1263

24. Jurickova I, Collins MH, Chalk C, Seese A, Bezold R, Lake K, von Allmen D, Frischer JS, Falcone RA, Trapnell BC, Denson LA (2013) Paediatric Crohn disease patients with stricturing behaviour exhibit ileal granulocytemacrophage colony-stimulating factor (GM-CSF) autoantibody production and reduced neutrophil bacterial killing and GM-CSF bioactivity. Clin Exp Immunol 172(3):455-465. doi:10.1111/cei.12076

25. Goldstein Jl, Kominsky DJ, Jacobson N, Bowers B, Regalia K, Austin GL, Yousefi M, Falta MT, Fontenot AP, Gerich ME, Golden-Mason L, Colgan SP (2011) Defective leukocyte GM-CSF receptor (CD116) expression and function in inflammatory bowel disease. Gastroenterology 141(1):208-216. doi:10.1053/j.gastro.2011.03.060

26. Han X, Gilbert S, Groschwitz K, Hogan S, Jurickova I, Trapnell B, Samson C, Gully $J$ (2010) Loss of GM-CSF signalling in non-haematopoietic cells increases NSAID ileal injury. Gut 59(8):1066-1078. doi:10.1136/gut.2009.203893

27. Nylund CM, D'Mello S, Kim MO, Bonkowski E, Dabritz J, Foell D, Meddings J, Trapnell BC, Denson LA (2011) Granulocyte macrophage-colony-stimulating factor autoantibodies and increased intestinal permeability in Crohn disease. $J$ Pediatr Gastroenterol Nutr 52(5):542-548. doi:10.1097/MPG.0b013e3181fe2d93

28. Uchida K, Beck DC, Yamamoto T, Berclaz PY, Abe S, Staudt MK, Carey BC, Filippi MD, Wert SE, Denson LA, Puchalski JT, Hauck DM, Trapnell BC (2007) GM-CSF autoantibodies and neutrophil dysfunction in pulmonary alveolar proteinosis. N Engl J Med 356(6):567-579. doi:10.1056/NEJMoa062505

29. Uchida K, Nakata K, Carey B, Chalk C, Suzuki T, Sakagami T, Koch DE, Stevens C, Inoue Y, Yamada Y, Trapnell BC (2014) Standardized serum GM-CSF autoantibody testing for the routine clinical diagnosis of autoimmune pulmonary alveolar proteinosis. J Immunol Methods 402(1-2):57-70. doi:10.1016/j.jim.2013.11.011

30. Däbritz J, Bonkowski E, Chalk C, Trapnell BC, Langhorst J, Denson LA, Foell D (2013) Granulocyte macrophage colony-stimulating factor auto-antibodies and disease relapse in inflammatory bowel disease. Am J Gastroenterol 108(12):1901-1910. doi:10.1038/ajg.2013.360

31. Bonneau J, Dumestre-Perard C, Rinaudo-Gaujous M, Genin C, Sparrow M, Roblin X, Paul S (2015) Systematic review: new serological markers (antiglycan, anti-GP2, anti-GM-CSF Ab) in the prediction of IBD patient outcomes. Autoimmun Rev 14(3):231-245. doi:10.1016/j.autrev.2014.11.004

32. Schwarzmaier D, Foell D, Weinhage T, Varga G, Dabritz J (2013) Peripheral monocyte functions and activation in patients with quiescent Crohn's disease. PLoS One 8(4):e62761. doi:10.1371/journal.pone.0062761

33. Däbritz J, Chalinor HV, Menheniott TR, Giraud AS, Judd LM (2013) Antiinflammatory effects of altered gp130 STAT3 signaling and the role of myeloid-derived suppressor cells in DSS induced colitis. United European Gastroenterol J 1(1S):A121-A122. doi:10.1177/2050640613502899

34. Varol C, Vallon-Eberhard A, Elinav E, Aychek T, Shapira Y, Luche H, Fehling HJ, Hardt WD, Shakhar G, Jung S (2009) Intestinal lamina propria dendritic cell subsets have different origin and functions. Immunity 31(3):502-512. doi:10.1016/j.immuni.2009.06.025

35. Rivollier A, He J, Kole A, Valatas V, Kelsall BL (2012) Inflammation switches the differentiation program of Ly6Chi monocytes from antiinflammatory macrophages to inflammatory dendritic cells in the colon. J Exp Med 209(1): 139-155. doi:10.1084/jem.20101387

36. Ostanin DV, Kurmaeva E, Furr K, Bao R, Hoffman J, Berney S, Grisham MB (2012) Acquisition of antigen-presenting functions by neutrophils isolated from mice with chronic colitis. J Immunol 188(3):1491-1502. doi:10.4049/jimmunol.1102296

37. Haile LA, von Wasielewski R, Gamrekelashvili J, Kruger C, Bachmann O, Westendorf AM, Buer J, Liblau R, Manns MP, Korangy F, Greten TF (2008) Myeloid-derived suppressor cells in inflammatory bowel disease: a new immunoregulatory pathway. Gastroenterology 135(3):871-881. doi:10.1053/j.gastro.2008.06.032, 881 e871-875

38. Roth L, MacDonald JK, McDonald JW, Chande N (2012) Sargramostim (GM-CSF) for induction of remission in Crohn's disease: a Cochrane Inflammatory Bowel Disease and Functional Bowel Disorders systematic review of randomized trials. Inflamm Bowel Dis 18(7):1333-1339. doi:10.1002/ibd.22973

39. Hamilton JA (2015) GM-CSF as a target in inflammatory/autoimmune disease: current evidence and future therapeutic potential. Expert Rev Clin Immunol 11(4):457-465. doi:10.1586/1744666X.2015.1024110
40. Griseri T, Arnold IC, Pearson C, Krausgruber T, Schiering C, Franchini F, Schulthess J, McKenzie BS, Crocker PR, Powrie F (2015) Granulocyte macrophage colony-stimulating factor-activated eosinophils promote interleukin-23 driven chronic colitis. Immunity 43(1):187-199. doi:10.1016/j.immuni.2015.07.008

41. Hamilton JA (2008) Colony-stimulating factors in inflammation and autoimmunity. Nat Rev Immunol 8(7):533-544. doi:10.1038/nri2356

42. Dranoff G (2011) Granulocyte-macrophage colony stimulating factor and inflammatory bowel disease: establishing a connection. Gastroenterology 141(1):28-31. doi:10.1053/j.gastro.2011.05.023

\section{Submit your manuscript to a SpringerOpen ${ }^{\odot}$ journal and benefit from:}

- Convenient online submission

- Rigorous peer review

- Immediate publication on acceptance

- Open access: articles freely available online

- High visibility within the field

- Retaining the copyright to your article

Submit your next manuscript at springeropen.com 